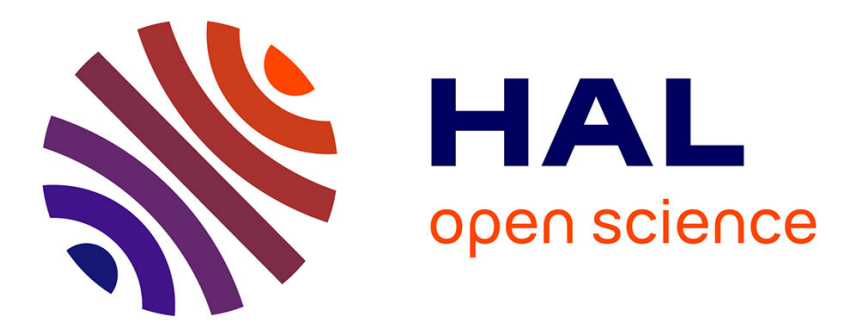

\title{
Contemplating Relativistic and Warp Speeds: Feasible Matter/Anti-Matter Propulsion and the Novel Theoretical Possibility of Attaining Warp Speeds
}

Mark Pickrell

\section{- To cite this version:}

Mark Pickrell. Contemplating Relativistic and Warp Speeds: Feasible Matter/Anti-Matter Propulsion and the Novel Theoretical Possibility of Attaining Warp Speeds. 2021 American Association of Aeronautics and Astronautics ASCEND Conference, Nov 2021, Las Vegas, NV, United States. hal-03477935

\section{HAL Id: hal-03477935 \\ https://hal.science/hal-03477935}

Submitted on 13 Dec 2021

HAL is a multi-disciplinary open access archive for the deposit and dissemination of scientific research documents, whether they are published or not. The documents may come from teaching and research institutions in France or abroad, or from public or private research centers.
L'archive ouverte pluridisciplinaire HAL, est destinée au dépôt et à la diffusion de documents scientifiques de niveau recherche, publiés ou non, émanant des établissements d'enseignement et de recherche français ou étrangers, des laboratoires publics ou privés. 


\title{
Contemplating Relativistic and Warp Speeds: Feasible Matter/Anti-Matter Propulsion and the Novel Theoretical Possibility of Attaining Warp Speeds
}

\author{
M. Pickrell ${ }^{1}$ \\ Virtuoso Surgical, Inc. \\ 5701 Old Harding Pike, Suite 200 \\ Nashville, TN 37205 \\ USA
}

\begin{abstract}
In Einstein's general theory of relativity, it is posited that speeds greater than the speed of light are not attainable. In recently published mathematical work by Dr. Eric Lentz at the Gottingen Institute for Astrophysics in Germany (following on the theoretical work of Miguel Alcubierre and others), it is theorized that speeds greater than the speed of light are possible. The energies necessary to achieve such speeds are, based on Dr. Lentz' work, monumental, and the physical mechanisms for achieving such speeds remain, at this point, speculative. With matter/anti-matter propulsion as a potentially feasible mechanism for approaching the speed of light, however, the possibility for acceleration toward warp speeds at the margin envelope is conceivable. Further theoretical work is needed to solve the Einstein field equations and, for plasma solitons, the Maxwell field equations, at the margin envelope of warp speed, assuming that matter/anti-matter propulsion can allow objects to approach the speed of light.
\end{abstract}

\section{Introduction}

This paper is prepared for the 2021 ASCEND Conference, TECH.EXPL-07 (Enabling Technologies: Miscellaneous). The purpose of the paper is to describe recent theoretical mathematical work performed by Dr. Erik Lentz at Gottingen Institute for Astrophysics in Germany (Dr. Lentz is now with JDSAT, Inc. in McLean, Virginia) theorizing the possibility of attaining speeds in space greater than the speed of light (i.e., "warp" speeds) using known energies and matter. The theoretical work of Dr. Lentz and his predecessors is intriguing, because it challenges one of Einstein's suppositions that speeds faster than the speed of light are not attainable. Coupled with the possibility of relativistic speeds (i.e., speeds that are a significant fraction of the speed of light) using matter anti-matter propulsion, advanced by the author in 2020, the possibility of attaining warp speeds as contemplated and calculated by Dr. Lentz is particularly intriguing for further theoretical work. Further work regarding energies at the margin envelope of the speed of light should be undertaken, and consideration of propulsion technologies that can transverse the warp barrier (which may be impossible at this time) should be undertaken in due course.

\footnotetext{
${ }^{1}$ General Counsel \& Chief Administrative Officer, Virtuoso Surgical, Inc.; AIAA Member.
} 


\section{Relativistic Speeds}

In 2020, based on experimental results of Hui Chen and others at the Lawrence Livermore Laboratory and Eve Stenson and others at the Max Planck Institute for Plasma Physics, the feasibility of attaining speeds at a significant fraction (even approaching) the speed of light, using matter/anti-matter annihilation for propulsion, was proposed by the author.[1] The idea of the matter/anti-matter propulsion system is to generate pairs of electrons and positrons using a petawatt laser striking a gold (or other) substrate, and then channeling those pairs of particles to an annihilation chamber where they can annihilate and generate thrust via the resulting $511-\mathrm{keV}$ gamma rays and the Compton Effect.

Because the "exhaust" of a matter/anti-matter propulsion system is composed of gamma rays traveling at the speed of light, the maximum theoretical speed of a matter/anti-matter system is the speed of light.

\section{A. Background -- The General Theory}

\section{Warp Speeds}

In his general theory of relativity, Albert Einstein theorized, in his terms, "invariance" of the speed of light as a constant of the universe (which, in his cosmology, was composed of four dimensions: the three conventional dimensions of space recognizable to us, and a fourth dimension, time, that operates with the other dimensions to constitute "spacetime", which can be understood as possessing a curved nature). Within spacetime, Einstein theorized that objects' location and momentum can be approximately defined using 16 field equations, generally known as the Einstein Field Equations.[2]

\section{B. Solving the Einstein Field Equations for Speeds Greater than the Speed of Light}

Since Einstein published his theory of relativity, solutions to the Einstein Field Equations have been determined by numerous theorists, such as G. Ellis and M. Madsen, for given, localized objects within a dynamic system.[3]

One of the theorists to calculate an approximate solution for achieving speeds greater than the speed of light is Miguel Alcubierre, who in 1994 theorized that an object (such as a spacecraft) could, in theory, occupy a "warp bubble" in which a single wave of spacetime can theoretically "carry" the object at speeds exceeding the speed of light.[4] One of the issues with Alcubierre's solutions for achieving speeds greater than the speed of light was the requirement that the energies involved would have to be negative energies, or the materials involved would have to be exotic in nature.[5]

Recently, Dr. Erik Lentz published an article in the Journal of Classical \& Quantum Gravity following on the work of Alcubierre, Van Den Broek, and others.[6] In his article, Dr. Lentz solves the Einstein Field Equations for a plasma soliton (it is useful to think of a soliton for this purpose as a single wave in spacetime) using a hyperbolic curvature for the curvature of spacetime. With his approach, Dr. Lentz was able to calculate that positive energies and known matter can, in theory, create a soliton wave, with a central "payload," that is capable of moving at speeds greater than the speed of light.

The mathematical work being formed to theorize speeds greater than light has shown a steady improvement -from Alcubierre to Lentz -- toward theoretical practicability. Whether additional theoretical work can lead to likely practicability will need to be demonstrated over (much) time. 


\section{Theoretical Objects to Pursue}

With the idea of a feasible matter/anti-matter propulsion system in play, which could, in theory, allow a craft to attain relativistic speeds and then to approach the speed of light, further theoretical work, following on Dr. Lentz' work (and his predecessors), should be pursued. In particular, with regard to, for example, a craft that weighs approximately $10,000 \mathrm{~kg}$, that has accelerated to $99 \%$ of the speed of light, how much energy would be required to accelerate that craft to $101 \%$ of the speed of light (assuming that a mechanism to exceed the speed of light exists)? In his article, Dr. Lentz anticipated,

One could self-consistently simulate the creation, propagation, and dismantlement phases of a soliton at both sub- and superluminal speeds. Other directions include further optimizations of the solutions over the energy requirements and other trade-offs, the broadening of the soliton geometry to incorporate a "payload" in the soliton's central region, and studying the challenges of horizon formation when transitioning to superluminal speeds.

In due course (which may be many, many years), a conceivable mechanism for surpassing the speed of light may be theorized. If so, then, one day, it may be possible to test Dr. Lentz' hypothesis. Before then, however, if matter/antimatter propulsion is pursued, and is feasible, many aspects of the General Theory can be tested, including whether there is any difference between gravitational inertia and the inertia of momentum, as relativistic speeds are achieved, on the curvature of spacetime; time dilation; and the invariance of the speed of light in a vacuum. With these aspects of the general theory tested, the suppositions underlying Alcubierre's, Van Den Broek's, Lentz's, and their contemporaries' mathematical work, can be evaluated and possibly refined.

\section{Conclusion}

Based on the recent work of Dr. Lentz, the theoretical possibility of warp speeds - speeds greater than the speed of light - is being enhanced. The energies necessary to attain such speeds are, based on Dr. Lentz' work, monumental, and the physical mechanism for achieving such speeds is, at this point in time, speculative. The possibility of approaching the speed of light via matter/anti-matter propulsion currently also exists, however, therefore the marginal energy required to break the warp barrier merits further theoretical \& mathematical work. In addition, the physical propulsion mechanism capable of generating speeds greater than the speed of light merits contemplation over time. Finally, one of the benefits of pursing relativistic speeds, using matter/anti-matter annihilation, will be to test and understand aspects of Einstein's General Theory in practice.

\section{References}

[1] M. Pickrell, "The feasibility of matter/anti-matter propulsion for generating relativistic speeds in space," J. Space Explor. 9(2): $162(2020)$.

[2] R. Adler, et al., Introduction to General Relativity (2d ed.), McGraw-Hill, New York (1975).

[3] G. Ellis \& M. Madsen, "Exact scalar field cosmologies,” J. Class. Quant. Gravity 8: 667-76 (1991).

[4] M. Alcubierre, “The warp drive: hyper-fast travel within general relativity,” J. Class. Quant. Gravity 11 (5): L73-L77 (1994).

[5] C. Van Den Broek, “A 'warp drive' with more reasonable total energy requirements,” J. Class. Quant. Gravity 16 (12): 3973 (1999).

[6] E. Lentz, "Breaking the warp barrier: hyper-fast solitons in Einstein-Maxwell plasma theory," J. Class. Quant. Gravity 38(7): 075015 (2021). 